\title{
Possibilistic Approach for Travel Time Reliability Evaluation
}

\author{
Rajesh S. Prabhu Gaonkar \\ School of Mechanical Sciences, \\ Indian Institute of Technology Goa (IIT Goa), \\ Farmagudi, Ponda, 403401, Goa, India. \\ Corresponding author: rpg@iitgoa.ac.in \\ Akshay V. Nigalye \\ Mechanical Engineering Department, \\ Goa College of Engineering (affiliated to Goa University), \\ Farmagudi, Ponda, 403401, Goa, India. \\ E-mail: avn@gec.ac.in \\ Sunay P. Pai \\ Institute of Maritime Studies, \\ Vasco - Da-Gama, Goa, India. \\ E-mail: sunaypai@imsgoa.in
}

(Received April 25, 2020; Accepted June 6, 2020)

\begin{abstract}
Travel time estimation \& reliability evaluation of any means of transportation in every type of travel mode- land, rail, sea and air has been of immense interest of the researchers; primarily due to growing economic concern in the field of logistics \& passenger movement. In situations like quantitative data inaccessibility or data imprecision, fuzzy set based possibilistic approach is recognized as a practical choice in obtaining the reliability estimates. This paper proposes and advocates possibilistic approach for travel time reliability computation of any type transportation vehicle under fuzzy type of data. The proposed approach is a novel way of computing the travel time \& obtaining the related reliability value. Initially, the paper proposes the general methodology for travel time reliability evaluation. Individual travel time components of a transportation vehicle are considered as fuzzy; as a result, travel time is modelled as a fuzzy variable. Travel time reliability of a transportation vehicle has been defined with the help of possibilistic measures. The proposed procedure is then demonstrated with an application to marine vessel carrying the bulk. After illustration of the proposed methodology, sensitivity analysis is carried out. The paper ends with the comments on comparative features of the three cases.
\end{abstract}

Keywords- Travel time, Possibilistic reliability, Possibility index, Fuzzy set, Maritime transportation system.

\section{Introduction}

Reliability of every transportation system is a multifaceted and complex issue, which involves numerous factors that are fluctuating in reality. An investigation of such systems involves several independent and dependent variables in entire transportation network. Competitive, cost-effective and optimized transportation cover some of the issues in reliable and safe travel. In recent years, several papers addressing transport network reliability and recognizing it as a main concern for potential investigations have been published (Nicholson and Dantas, 2004; Sumalee and Kurauchi, 2006). In the performance analysis of transportation systems, uncertainty in travel time has been a popular choice of researchers (Senna, 1994; Noland and Polak, 2002; Ettema and Timmermans, 2006). Chang (2010) suggested an approach of assessing travel time reliability in transport appraisal. He considered the gap between actual and planned journey times for the quantification, 
International Journal of Mathematical, Engineering and Management Sciences

Vol. 6, No. 1, 223-243, 2021

https://doi.org/10.33889/IJMEMS.2021.6.1.014

subject to the differing characteristics of road and rail usage. Prabhu Gaonkar et al. (2011) presented a methodology of maritime transportation system reliability evaluation by the application of fuzzy sets and fuzzy logic techniques. Prabhu Gaonkar et al. (2013a) formulated maritime transportation reliability problem in mathematical form and demonstrated its solution methodology using possibilistic programming. Prabhu Gaonkar et al. (2013b) proposed two fuzzy reliability-based models for estimation of maritime transportation reliability.

It is also observed that travel time reliability has been researched with core focus on land transportation (Sanchez-Silva et al., 2005; Wu et al., 2008; Vanajakshi et al., 2009; El Faouzi et al., 2010). Batley (2007) studied issues related to travel time reliability and scheduling aspects. Some other investigations in travel time, reliability forecast, simulation and benchmarking include (Noland et al., 1998; Nam et al., 2005; van Lint, 2008; van Lint et al., 2008; Margulici and Ban, 2008; Kwong et al., 2010; Yang et al., 2010). Tzannatos (2005) assessed technical reliability of the Greek coastal passenger fleet. Li et al. (2010) reviewed critically the modeling frameworks and empirical measurement scenarios used to obtain willingness to pay for improved travel time reliability and suggested new directions for ongoing research.

The paper (Bell, 2000) outlined a novel approach using game theory to the measurement of network reliability. In Clark and Watling (2005), a technique is developed for estimating the probability distribution of total network travel time with respect to normal everyday variations in the travel demand matrix over a road traffic network. Fan and Nie (2006) studied routing problem of maximizing travel time reliability by modeling it in the time adaptive route choice framework. Fosgerau and Karlstrom (2010) estimated the value of reliability in the scheduling of an activity of random duration, such as travel under congested conditions. The paper Al-Deek and Emam (2006) presented method to compute travel time reliability in transportation networks with multistate and dependent link failures. Janic (2003) studied about performance of air transportation whereas Hollander and Liu (2008) illustrated how to obtain the travel time distribution with series of simulated experiments. Innamaa (2005) proposed prediction of travel time using neural networks; whereas Zheng and McDonald (2009) estimated the travel time using fuzzy clustering method. In addition, the real travel time data measurement errors and summarized the advantages of using fuzzy approach.

In recent publications, $\mathrm{Li}$ et al. (2019) extended travel time reliability from the perspective of predictability based on the Lempel-Ziv algorithm that provides an upper bound of travel time predictability based on historical data. Ghader et al. (2019) used cumulative prospects theory for modelling the travel time reliability on mode choice and its extensions. $\mathrm{Li}$ (2019) used travel time reliability ratio (TTRR) defined as the ratio of the value of travel time variability to the value of travel time which is an important metric for measuring such reliability. He measured and demonstrated with practical example the travel time reliability and risk using nonparametric approach. Chen and Fan (2020) analysed travel time distribution based on different travel time reliability patterns. Saedi et al. (2020) estimated network vehicle travel time reliability with network partitioning. Wu et al. (2020) estimated travel time of ships in narrow channel based on AIS data.

As seen from the literature survey, among the few investigations that are carried out related to the travel time reliability issues; the primary viewpoint is the stochastic nature of the variables. Furthermore, the researchers have not taken into consideration the possibilistic aspect for modelling the travel time and its components. The main intention of this paper is to present a general approach 
International Journal of Mathematical, Engineering and Management Sciences

Vol. 6, No. 1, 223-243, 2021

https://doi.org/10.33889/IJMEMS.2021.6.1.014

to evaluate the travel time reliability of any transportation vehicle with focus on fuzziness or possibilistic nature of the input variables. Fuzzy set-based techniques are applied to formulate the various situations under which reliability computation would be necessitated. The organization of the paper is as follows: Firstly, preliminaries such as concept of possibilistic theory and possibility measures are presented which are required for defining and evaluating the travel time reliability. Then, travel time components and the concept of fuzzy travel time is introduced. This is followed by the definition of possibilistic reliability of a general transportation vehicle. The general computation procedure is explained next in Section 4. This section also states the method of aggregation of expert opinions and makes few comments on elicitation of membership function by analyst. The proposed methodology is illustrated in Section 5 with an application to marine vessel transportation. Three situations or cases are formulated depending on the nature of the travel time and scheduled time. The illustration of the cases is shown with suitable input data set and after that, sensitivity analysis is carried out on the fuzziness associated with the variables. Features of the three cases are as well presented at the end of Section 5. Finally, the paper ends with summary and conclusion.

\section{Possibility- Concept and Measures}

Reliability is an important quality performance measure of every transportation system. Estimation of travel time reliability value is carried out using possibilistic measures in this paper. In recent years, possibility approach is admired due to its ability to model in an environment or system where there is less and/ or imprecise information or data. Possibility theory, a variant of fuzzy set theory is one method that does not require detailed statistical data. Fuzzy set theory provides a way of dealing with imprecise or scarce information computationally. As a result, use of fuzzy and possibilistic reliability concept has been growing over the years (Li et al., 2000; Jiang and Chen, 2003; Wu, 2004; Marano and Quaranta, 2010). In some recent research related to fuzzy reliability, general fuzzy multi-state system and component models have been proposed (Ding and Lisnianski, 2008a; Ding et al., 2008b; Liu et al., 2008), in which the state probability or/ and the state performance level of a system component can be treated as fuzzy values. Few recent papers in mathematical programming formulations with aleatory and epistemic uncertainty in the reliability assessment are Huang and Zhang (2009) and Zhang and Huang (2010).

Parameter uncertainties are classified into two distinct types: aleatory and epistemic uncertainty. Aleatory uncertainty is irreducible or inherent uncertainty and it arises as variability resulting from heterogeneity or stochasticity. It is due to randomness i.e. due to variability phenomena. Epistemic uncertainty is a subjective or reducible uncertainty that stems from lack of knowledge and data. This uncertainty arises as partial ignorance resulting from systematic measurement error or subjective uncertainty. It is due to incompleteness i.e. when information regarding the parameter values is merely lacking. This type of uncertainty is dealt by possibility theory that uses possibility measures which are directly connected with the fuzzy sets (Klir et al., 1997). For requisite elementary information of fuzzy set concepts and in detail possibilistic measures, readers may refer Klir et al. (1997); Inuiguchi and Ramik (2000). Following paragraphs present indices defined by possibility measures that are applied for possibilistic reliability computation in this paper.

Let $\psi$ be a possibilistic variable and $\tilde{A}$ and $\tilde{B}$ are fuzzy sets that need to be compared. Under possibilistic distribution $\mu_{A}$ of a possibility variable $\psi$, possibility measure of the event that $\psi$ is in fuzzy set $\tilde{B}$ is defined as Inuiguchi and Ramik (2000). 
International Journal of Mathematical, Engineering and Management Sciences

Vol. 6, No. 1, 223-243, 2021

https://doi.org/10.33889/IJMEMS.2021.6.1.014

$\Pi_{A}(\tilde{B})=\sup \min \left(\mu_{A}(r), \mu_{B}(r)\right)$

where $\mu_{B}$ is the membership function of the fuzzy set $\tilde{B}$ and $r$ represents variable domain values (on $X$-axis). $\Pi_{A}(B)$ evaluates to what extent it is possible that $\psi$ restricted by the possibility distribution $\mu_{A}$ is in the fuzzy set $\tilde{B}$.

Now to compare a fuzzy set with the crisp value, let $B$ be non-fuzzy i.e. crisp set of real numbers, which is not greater than $g . B=(-\infty, g]$. Possibility index is then defined as Inuiguchi and Ramik (2000).

$$
\begin{aligned}
\operatorname{Pos}(\psi \leq g) & =\Pi_{A}((-\infty, g]) \\
& =\sup \left\{\mu_{A}(r) \mid r \leq g\right\}
\end{aligned}
$$
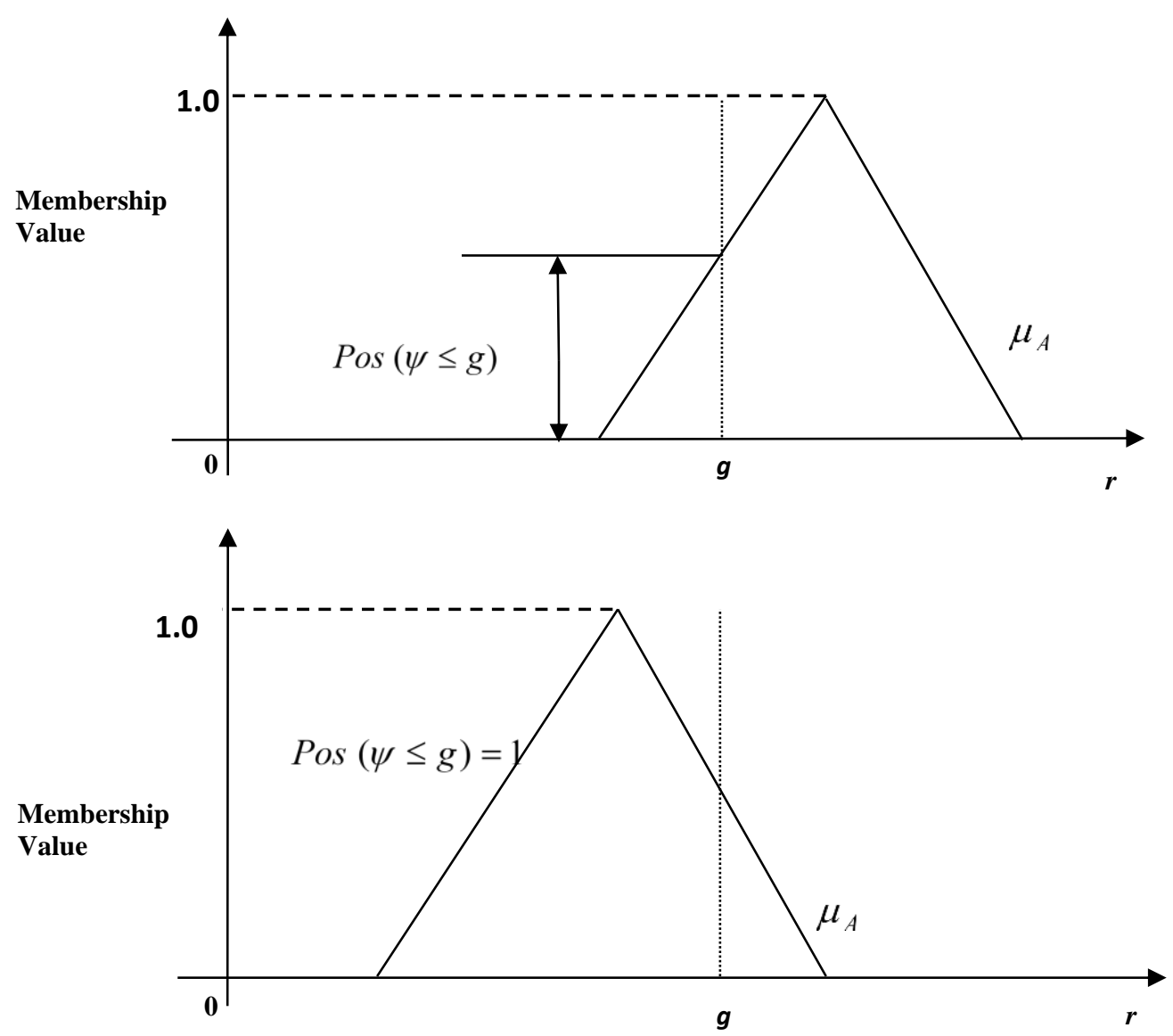

Figure 1. Possibility degree of $(\psi \leq g)$ 
International Journal of Mathematical, Engineering and Management Sciences

Vol. 6, No. 1, 223-243, 2021

https://doi.org/10.33889/IJMEMS.2021.6.1.014

$\operatorname{Pos}(\psi \leq g)$ show the possibility degree to what extent $\psi$ is not greater than $g$. This index for Triangular Fuzzy Number (TFN) and crisp value $g$ is shown in Figure 1.

Two TFN's can as well be compared based on the exceedance possibility given in the literature (Negi and Lee, 1993; Iskander, 2002). If $\tilde{A}$ and $\tilde{B}$ are two TFN's, where $\tilde{A}=\left\{a, a_{0}, \bar{a}\right\}$ and

$$
\begin{aligned}
& \tilde{B}=\left\{b, b_{0}, \bar{b}\right\} \text {, then the possibility that }\{\tilde{B} \leq \tilde{A}\} \text { is given as: } \\
& \operatorname{Pos}(\tilde{B} \leq \tilde{A})=\theta \quad ; b_{0} \leq a_{0} \\
& =\frac{(\bar{a}-\underline{b}) \theta}{\left(\bar{a}-a_{0}\right)+\left(b_{0}-b\right)} \quad ; b_{0} \geq a_{0}, \quad b \leq \bar{a} \\
& =0 \quad ; b \geq \bar{a}
\end{aligned}
$$

where $\theta$ is the maximum value of the membership function of $\tilde{A}$, i.e. when $a=a_{0}$.

\section{Travel Time Reliability- Possibilistic View 3.1 Fuzziness in Travel Time}

A range of times constitute the transportation vehicle travel time. There are various components of the travel time that would dominate the total travel time. E.g. total travel time of an aircraft consist of preparation time before departure, waiting time, takeoff time, travel time landing time etc. All the components would dominate the total travel time. If actual departure time is missed by the aircraft, further modification in schedule would be demanded, which in turn would hinder the total travel time. As a result, there would be delay in reaching at the destination airport.

Travel times are uncertain and deviate in spite of proper precautions. It is difficult to predict due to the variations of many kinds. Uncertainties in times can be of randomness type or may relate to imprecision or vagueness. Statistical treatment is the distinct option to estimate the vessel travel time in case proper documented data or information is available. However, such a thing is seldom; and other approaches are necessitated to estimate the same.

Gathering the precise data for calculating the values of the travel time components is not a trivial task. This issue, in the absence of the statistical data, can be modelled well using possibilistic approach. The components of travel time of every transportation vehicle are imprecise and the information about these times can be gathered from the personnel working closely with the particular mode of transportation. Obviously, the information would be based on the personnel's opinions and judgments and is of subjective or fuzzy type.

As all individual travel time components are regarded as fuzzy, travel time is modelled here as a fuzzy variable. The travel time of transportation vehicle is sum of all its component times, which are individually fuzzy in nature: 
International Journal of Mathematical, Engineering and Management Sciences

Vol. 6, No. 1, 223-243, 2021

https://doi.org/10.33889/IJMEMS.2021.6.1.014

$\tilde{T}=\tilde{T}_{1}+\tilde{T}_{2}+\tilde{T}_{3}+\tilde{T}_{4}+\cdots \cdots \cdots \cdots+\tilde{T}_{m}$

' $\sim$ ' is used to denote a fuzzy variable, $\widetilde{\boldsymbol{T}}$ is the total travel time and $\widetilde{\boldsymbol{T}}_{\boldsymbol{m}}(\boldsymbol{m}=1,2,3, \ldots \ldots$ and so on) is the travel time components. The nature of membership functions of the fuzzy component times and consequently travel time may differ. This paper considers triangular and symmetric Gaussian membership functions for the same. It may also be noted that all the travel time components are assumed as independent fuzzy variables.

\subsection{Possibilistic Reliability}

The term 'reliability of a transportation vehicle' is described in this paper as a 'possibility' that the transportation vehicle under consideration reach the intended destination in scheduled time to accomplish the intended mission. The intended mission would differ and it depends on the type of the transportation vehicle i.e. cargo plane, passenger plane, oil tanker, goods train or defence ship etc. Each transportation vehicle has departure-arrival schedules. In view of the same, reliability measure is concerned with the transportation vehicle reaching as per the scheduled time at the destination. Accomplishment of mission with fulfilment of the assigned task such as timely delivery of cargo or oil or passenger arrivals at destination port as per their plans is a significant feature of the reliability definition. Accordingly, possibilistic reliability of a transportation vehicle is

$R=\operatorname{Pos}(\tilde{T} \leq t) ; t \geq 0$

where 'Pos' stand for possibility. The right-hand side term of 'lesser than or equal to' sign i.e. time ' $t$ ' is the scheduled or planned time at which transportation vehicle is supposed to reach at the destination to complete the assigned mission objective(s). Equation (5) considers that even if there is negative side variability meaning the vehicle reaches early or before the scheduled time, it is considered as a part of reliability definition. In fact, the issue whether an early arrival to be considered as part of reliability specification or not would necessitate cost-benefit study and analysis. Transportation vehicle reaching early at the destination is considered as on-time arrival in this paper. This is due the fact that early arrivals are regarded as benefits because absolute journey time decreases (Chang, 2010).

This paper considers time ' $t$ ' as crisp (i.e. non-fuzzy) as well as a fuzzy variable. The illustration section of the paper brings out the suitable examples to demonstrate this aspect. If non-fuzzy values for variable ' $t$ ' are $10,20,30, \ldots$ and so on (in appropriate time units); then for fuzzy case, they are modelled as 'about 10', 'about 20', 'about 30 ', ... etc. Suitable membership functions have been considered for modelling such ambiguity type of uncertainty in the scheduled time values.

\section{Computation Procedure}

\subsection{Calculation Technique}

Evaluation of equation (4) needs fuzzy arithmetic process. This would differ depending upon the

type of membership function considered. If $\tilde{A}=\left\{a, a_{0}, \bar{a}\right\}$ and $\tilde{B}=\left\{b, b_{0}, \bar{b}\right\}$ are two TFN's, then $\tilde{A}(+) \tilde{B}=\left\{a+b, a_{0}+b_{0}, \bar{a}+\bar{b}\right\}$, which is also a TFN (Klir et al., 1997). Gaussian fuzzy sets are another way to represent fuzzy numbers and are convenient and natural to work with in real space. When two Gaussian fuzzy numbers are added, the universal space almost doubles. The resulting 
International Journal of Mathematical, Engineering and Management Sciences

Vol. 6, No. 1, 223-243, 2021

https://doi.org/10.33889/IJMEMS.2021.6.1.014

fuzzy set obtained by adding two Gaussian membership functions, is also Gaussian fuzzy set, with mean and standard deviation equal to the sum of the means and standard deviations of the original membership functions being added.

Subsequently, equation (5) can be evaluated to obtain possibilistic reliability of marine vessel using the possibility measures as explained in earlier section. The analysis procedure would differ depending upon the membership function of travel time and nature of scheduled time. Whether scheduled time (right hand side constraint of equation (5)) is fuzzy or non-fuzzy (crisp) mainly becomes the major aspect in the possibilistic reliability computation process.

\subsection{Aggregation of Expert Opinions}

In implementation of possibilistic uncertainty techniques, opinions expressed by the domain experts play a significant role. Various methods can be found in literature to combine the expert's responses. This section briefly presents two such techniques; the first one for TFN's and the other related to Gaussian fuzzy numbers.

For aggregating the opinions expressed in TFN, a technique suggested by Cai (1996), and further applied by Verma et al. (2006), is quite well accepted. This method uses responses of ' $n$ ' number of human experts assigning values to the variable in symmetric TFN format. The individual expert's responses be: $E^{(i)}=\left(D_{m}{ }^{(i)}-d^{(i)}, D_{m}{ }^{(i)}, D_{m}{ }^{(i)}+d^{(i)}\right) ; i=1,2, \ldots ., n$. The combined expert group's opinion is: $O=\left(O_{m}-\right.$ diff $, O_{m}, O_{m}+$ diff $)$, which is as well a symmetric TFN. The parameters $O_{m}$ and diff of the resulting TFN ' $O$ ' are determined using the following:

$$
\begin{aligned}
& O_{m}=\frac{\min _{1 \leq i \leq n} D^{(i)}+\max _{1 \leq i \leq n} D_{m}{ }^{(i)}}{2} \\
& \text { diff }=\frac{1}{n} \cdot \sum_{i=1}^{n} d^{(i)}
\end{aligned}
$$

The second aggregation method is reasonably practical when the opinions expressed are imprecise and are modelled as Gaussian membership function. Gaussian functions are the most adequate choice of membership functions for representing uncertainties in measurement (Kreinovich, 1992). The procedure to obtain parameters of Gaussian membership function from expert's imprecise estimates is as follows: If for a quantity ' $X$ ', several experts (say ' $n$ ') give their estimates as: $a_{1}, a_{2}$, $a_{3}, \ldots \ldots \ldots, a_{n}$, and they estimate the precision of their estimates correspondingly as: $\delta_{1}, \delta_{2}, \delta_{3}, \ldots \ldots \ldots$, $\delta_{n}$, then the resulting membership function is given by:

$\mu(x)=e^{-\beta\left\{\frac{(x-a)^{2}}{\delta^{2}}\right\}} \quad ;$ for $\beta>0$

where $\delta=\frac{1}{\sqrt{\frac{1}{\delta_{1}^{2}}+\frac{1}{\delta_{2}^{2}}+\frac{1}{\delta_{3}^{2}}+\ldots+\frac{1}{\delta_{n}^{2}}}} \quad$ and $\quad a=\frac{\frac{a_{1}}{\delta_{1}^{2}}+\frac{a_{2}}{\delta_{2}^{2}}+\frac{a_{3}}{\delta_{3}^{2}}+\ldots+\frac{a_{n}}{\delta_{n}^{2}}}{\frac{1}{\delta_{1}^{2}}+\frac{1}{\delta_{2}^{2}}+\frac{1}{\delta_{3}^{2}}+\ldots+\frac{1}{\delta_{n}^{2}}}$

This theorem is proved by Kreinovich et al. (1992). The membership function $\mu(x)$ stated above is 
International Journal of Mathematical, Engineering and Management Sciences

Vol. 6, No. 1, 223-243, 2021

https://doi.org/10.33889/IJMEMS.2021.6.1.014

a symmetric Gaussian function with parameters: central value ' $a$ ', deviation (i.e. delta) ' $\delta$ ' and fuzziness parameter $\beta$. Values of $a$ and $\delta$ are obtained based on expert's responses and parameter $\beta$ is decided by the analyst involved in membership function elicitation process.

\subsection{Membership Function Elicitation}

The membership function of a fuzzy set or a fuzzy number is a generalization of the indicator function in classical set. It portrays measure of fuzziness. It represents the degree of truth as an extension of valuation. Conceptually degree of truth is distinct with that of probabilities. Membership functions can have different shapes such as Triangular, Trapezoid, Sigmoidally shaped, Gaussian, S-shaped, Z-shaped etc. The membership function is problem dependent and its shape must suit from the point of view of simplicity, convenience, and truth representation in the problem domain. Fuzzy sets are usually intended to model human expert's cognitive states and they can be determined from either simple or sophisticated elicitation procedures (Medasani et al., 1998; Inuiguchi et al., 2000; Pedrycz and Vukovich, 2002; Yu and $\mathrm{Wu}$, 2009). These membership functions are estimated and determined using elicitation methods based on one of the perspectives such as likelihood view, similarity view, utility view, measurement view and possibilistic view (Reventos, 1999). Few methods are described in (Klir et al., 1997), for determining the membership functions that are essentially based on direct methods of inquiry made on human experts in the problem domain and corrected using indirect methods. The methods have been also categorized as automatic methods, statistical methods and psychological methods, based on type and nature of data. Neural networks are also been used for estimation of membership functions (Halgamuge et al., 1995; Klir et al., 1997).

The selection of membership functions and choice of its parameters directly affect the performance of the model. The fuzziness parameters of membership functions i.e. spread in case of TFN and $\beta$ in case of Gaussian fuzzy numbers need to be selected by the analyst based on interaction with the experts who would be providing their opinion/ judgment regarding a particular model variable. For this, study of opinion pattern of the expert's associated with the problem under investigation is done and then parameter values are decided based on the elicited information.

\section{Case Illustration- Application to Marine Vessel}

The marine vessel carrying the bulk cargo between Incheon (on west coast of Korea) and Tokyo (Japan) has been considered here as an illustrative example. The various dominant components of the marine vessel's travel times that are considered for reliability computation are:

(i) Preparation time for departure at source harbor $\left(T_{p s h}\right)$

(ii) Waiting time subsequent to preparation at source harbor $\left(T_{w s h}\right)$

(iii) Navigation time at sea or ocean $\left(T_{n}\right)$

(iv) Delay time due to unforeseen events $\left(T_{d}\right)$

(v) Waiting time at sea or ocean before docking at destination harbor $\left(T_{w d h}\right)$

(vi) Docking time at destination harbor $\left(T_{d d h}\right)$

(vii) Clearance time at destination harbor $\left(T_{c d h}\right)$

All above individual travel time components are modelled as fuzzy variables. As a result, marine 
International Journal of Mathematical, Engineering and Management Sciences

Vol. 6, No. 1, 223-243, 2021

https://doi.org/10.33889/IJMEMS.2021.6.1.014

vessel's total travel time which is sum of all its component times, also becomes a fuzzy variable. Thus, fuzzy total travel time is:

$\tilde{T}=\tilde{T}_{p s h}+\tilde{T}_{w s h}+\tilde{T}_{n}+\tilde{T}_{d}+\tilde{T}_{w d h}+\tilde{T}_{d d h}+\tilde{T}_{c d h}$

All the marine vessel travel time components are considered as independent fuzzy variables with different membership functions. These variables are modelled using triangular and symmetric Gaussian membership functions.

Possibilistic theory has several variants and technique for possibilistic reliability computation could be established based on the nature of the data and the information available. The methodology proposed here is practically useful in the absence of ready statistical data or limitations of documentation, financial and other resources to acquire and maintain the necessary data. To illustrate the computation procedure, this section formulates three illustrative situations (cases) depending upon the nature of scheduled time constraint and the availability of fuzzy natured data related to previously listed components of travel time. First two cases model uncertainty in travel time components as TFN's; whereas in third case, imprecision in travel time components leading to Gaussian membership function have been considered. Constraint variable i.e. scheduled time of the marine vessel have been regarded as crisp in first and third case; and in second case, it is accepted as TFN. These are presented in subsections 5.1, 5.2 and 5.3 respectively. Subsection 5.4 presents the results of parameter sensitivity and subsection 5.5 offers comments on the three cases.

Opinions/ judgments of six experts well versed with all the operations of the marine vessel in the sector between Incheon and Tokyo are considered. Responses from the six experts have been obtained for the seven travel time components listed previously. Illustrative input data for the cases evaluations is as shown in Table 1.

Table 1. Input data set for travel time computation.

\begin{tabular}{|c|c|c|c|c|c|c|c|c|c|c|c|c|}
\hline \multirow{3}{*}{$\begin{array}{c}\text { Time } \\
\text { Compone } \\
\text { nts }\end{array}$} & \multicolumn{12}{|c|}{ Individual Expert's Opinion (in hours) } \\
\hline & \multicolumn{2}{|c|}{1} & \multicolumn{2}{|c|}{2} & \multicolumn{2}{|c|}{3} & \multicolumn{2}{|c|}{4} & \multicolumn{2}{|c|}{5} & \multicolumn{2}{|c|}{6} \\
\hline & $D_{m}^{(1)}$ & $d^{(1)}$ & $D_{m}^{(2)}$ & $d^{(2)}$ & $D_{m}^{(3)}$ & $d^{(3)}$ & $D_{m}^{(4)}$ & $d^{(4)}$ & $D_{m}^{(5)}$ & $d^{(5)}$ & $D_{m}^{(6)}$ & $d^{(6)}$ \\
\hline$\widetilde{T}_{p s h}$ & 3 & 1 & 5 & 2 & 4 & 2 & 4 & 1 & 3 & 1 & 6 & 3 \\
\hline$\tilde{T}_{w s h}$ & 2 & 1 & 1 & 1 & 1 & 1 & 2 & 1 & 3 & 2 & 1 & 1 \\
\hline$\tilde{T}_{n}$ & 64 & 5 & 59 & 3 & 60 & 5 & 66 & 4 & 62 & 7 & 63 & 6 \\
\hline$\tilde{T}_{d}$ & 8 & 3 & 6 & 3 & 11 & 4 & 9 & 3 & 12 & 4 & 11 & 3 \\
\hline$\tilde{T}_{w d h}$ & 6 & 2 & 3 & 2 & 3 & 2 & 5 & 2 & 4 & 2 & 3 & 2 \\
\hline$\tilde{T}_{d d h}$ & 1 & 1 & 2 & 1 & 1 & 1 & 3 & 1 & 3 & 1 & 2 & 1 \\
\hline$\tilde{T}_{c d h}$ & 3 & 2 & 4 & 2 & 3 & 1 & 4 & 2 & 2 & 1 & 4 & 3 \\
\hline
\end{tabular}

\subsection{Travel Time and its Components are TFN's, and Scheduled Time is Crisp}

Using the data from Table 1 and applying aggregation technique explained in earlier section, combined group decision for each travel time components is obtained. Table 2 gives the final TFN's for each time component. Subsequently, utilizing fuzzy arithmetic, TFN for travel time is obtained as: $\widetilde{T}=\left[\widetilde{T}_{L}, \widetilde{T}_{M}, \widetilde{T}_{R}\right]=[71.50,87.50,103.50]$. TFN of travel time is depicted in Figure 2. 
International Journal of Mathematical, Engineering and Management Sciences

Vol. 6, No. 1, 223-243, 2021

https://doi.org/10.33889/IJMEMS.2021.6.1.014

For assessment of possibilistic reliability of a marine vessel, possibilistic degree of $\psi \leq g$ i.e. $\tilde{T} \leq$ $t$ is found out. For example, for crisp scheduled time of 85 and travel time [71.50, 87.50, 103.50], the possibilistic degree turns out to be 0.8438 . This is as per the definition and depiction of possibility measure from Figure 1 . Therefore, possibilistic reliability for this combination is $84.38 \%$. For a range of scheduled times between 71.5 to 87.5 , values of possibilistic reliability have been tabulated in Table 3. Possibilistic reliability is 0 below 71.5, while it takes a value 1 above 87.5.

Table 2. Combined group decision TFN's of travel time components.

\begin{tabular}{|c|c|}
\hline Time Components & Combined Group Decision \\
\hline$\tilde{T}_{p s h}$ & {$[2.83,4.50,6.17]$} \\
\hline$\tilde{T}_{w s h}$ & {$[0.83,2.00,3.17]$} \\
\hline$\tilde{T}_{h}$ & {$[57.50,62.50,67.50]$} \\
\hline$\tilde{T}_{d}$ & {$[5.67,9.00,12.33]$} \\
\hline$\tilde{T}_{w d h}$ & {$[2.50,4.50,6.50]$} \\
\hline$\tilde{T}_{d d h}$ & {$[1.00,2.00,3.00]$} \\
\hline$\tilde{T}_{c d h}$ & {$[1.17,3.00,4.83]$} \\
\hline
\end{tabular}

Table 3. Possibilistic reliability values (First case).

\begin{tabular}{|c|c|}
\hline Scheduled Time & Possibilistic Reliability \\
\hline 71.5 & 0.0000 \\
\hline 72 & 0.0313 \\
\hline 73 & 0.0938 \\
\hline 74 & 0.1563 \\
\hline 75 & 0.2188 \\
\hline 76 & 0.2813 \\
\hline 77 & 0.3438 \\
\hline 79 & 0.4063 \\
\hline 80 & 0.4688 \\
\hline 82 & 0.5313 \\
\hline 83 & 0.5938 \\
\hline 84 & 0.6563 \\
\hline 85 & 0.7188 \\
\hline 86 & 0.7813 \\
\hline 87 & 0.8438 \\
\hline 87.5 & 0.9063 \\
\hline & 0.9688 \\
\hline
\end{tabular}


International Journal of Mathematical, Engineering and Management Sciences

Vol. 6, No. 1, 223-243, 2021

https://doi.org/10.33889/IJMEMS.2021.6.1.014

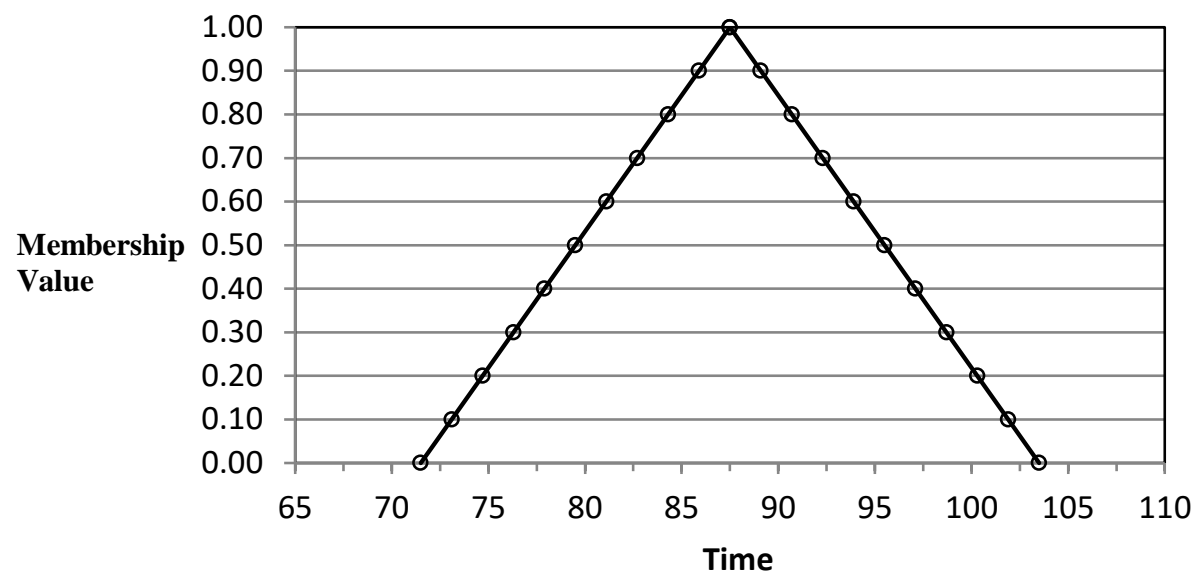

Figure 2. TFN of travel time.

\subsection{Travel Time, Its Components and Scheduled Time are All TFN's}

This case considers constraint variable i.e. scheduled time is non-crisp; more specifically as a TFN. The scheduled time having a value in the form of TFN i.e. $\tilde{t}=\left[\tilde{t}_{L}, \widetilde{t}_{M}, \tilde{t}_{R}\right]$ can be elucidated as the variable captivating a value 'about $t_{M}$ '; for example, 'about 65', 'about 70', 'about 86', and so on. As scheduled time is modelled as TFN, fuzziness, in other words, imprecision or spread of $10 \%$ on each side of centre value (i.e. $\tilde{t}_{M}$ where membership value is 1.0) have been assumed for getting the support set $\left[\tilde{t}_{L}, \tilde{t}_{R}\right]$ of the constraint variable. A wide range of fuzzy scheduled times have been considered as in earlier case as shown in Table 4. In addition, TFN for scheduled time is a normal fuzzy set i.e. maximum value of the membership function is unity. This leads to value of $\theta=1.0$ in equation (3). Same input data as in earlier case is considered here as well for estimation of travel time components and travel time. With $\widetilde{T}=[71.50,87.50,103.50]$, possibilistic reliability for various fuzzy scheduled times is computed as shown in Table 4. Figure 3 shows possibilistic reliability computation for one particular scheduled time $\tilde{t}=\left[\tilde{t}_{L}, \tilde{t}_{M}, \tilde{t}_{R}\right]=[70.2$, 78, 85.8]. When this TFN is compared with estimated $\tilde{T}$, possibility that $\tilde{T} \leq \tilde{t}$ is 0.6008 ; leading to reliability value of $60.08 \%$.

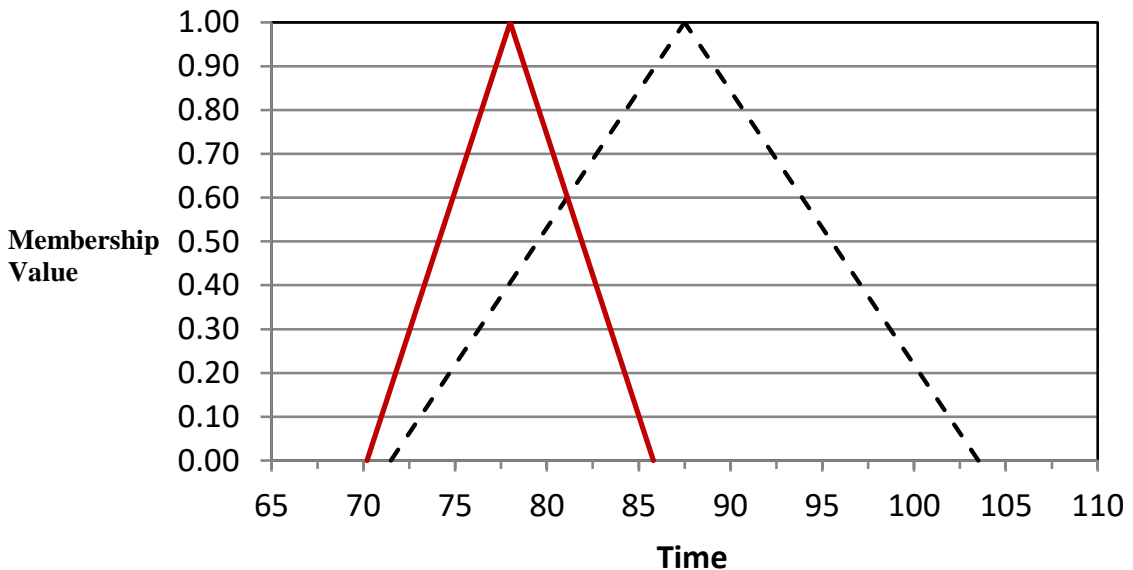

Figure 3. A Particular scheduled time TFN $[70.2,78,85.8]$ in comparison. 
International Journal of Mathematical, Engineering and Management Sciences

Vol. 6, No. 1, 223-243, 2021

https://doi.org/10.33889/IJMEMS.2021.6.1.014

Table 4. Possibilistic reliability values (Second case).

\begin{tabular}{|c|c|c|c|}
\hline \multicolumn{3}{|c|}{ Scheduled Time } & \multirow{2}{*}{$\begin{array}{c}\text { Possibilistic } \\
\text { Reliability }\end{array}$} \\
\hline$\tilde{t}_{L}$ & $\tilde{t}_{M}$ & $\tilde{t}_{R}$ & \\
\hline 58.5 & 65 & 71.5 & 0.0000 \\
\hline 59.4 & 66 & 72.6 & 0.0487 \\
\hline 60.3 & 67 & 73.7 & 0.0969 \\
\hline 61.2 & 68 & 74.8 & 0.1447 \\
\hline 62.1 & 69 & 75.9 & 0.1921 \\
\hline 63 & 70 & 77 & 0.2391 \\
\hline 63.9 & 71 & 78.1 & 0.2857 \\
\hline 64.35 & 71.5 & 78.65 & 0.3089 \\
\hline 64.8 & 72 & 79.2 & 0.3319 \\
\hline 65.7 & 73 & 80.3 & 0.3777 \\
\hline 66.6 & 74 & 81.4 & 0.4231 \\
\hline 67.5 & 75 & 82.5 & 0.4681 \\
\hline 68.4 & 76 & 83.6 & 0.5127 \\
\hline 69.3 & 77 & 84.7 & 0.5570 \\
\hline 70.2 & 78 & 85.8 & 0.6008 \\
\hline 71.1 & 79 & 86.9 & 0.6444 \\
\hline 72 & 80 & 88 & 0.6875 \\
\hline 72.9 & 81 & 89.1 & 0.7303 \\
\hline 73.8 & 82 & 90.2 & 0.7727 \\
\hline 74.7 & 83 & 91.3 & 0.8148 \\
\hline 75.6 & 84 & 92.4 & 0.8566 \\
\hline 76.5 & 85 & 93.5 & 0.8980 \\
\hline 77.4 & 86 & 94.6 & 0.9390 \\
\hline 78.3 & 87 & 95.7 & 0.9798 \\
\hline 78.75 & 87.5 & 96.25 & 1.0000 \\
\hline
\end{tabular}

\subsection{Travel Time and Its Components are Gaussian Fuzzy Numbers, and Scheduled Time is Crisp}

This case is rather different from earlier two cases, in the fact that it considers imprecision in the input data that is tackled using Gaussian concepts. Travel time components have been modelled as Gaussian fuzzy numbers and scheduled time is considered as a non-fuzzy value. Same data as in Table 1 is considered as opinions of the six experts about travel time components. However, as modelling is using Gaussian membership function and not a TFN, expert's estimates are $a_{i} ; i=1,2,3,4,5,6$ in place of $D_{m}^{(i)} ; i=1,2,3,4,5,6$. Correspondingly, the precision of six experts in their estimates are $\delta_{i} ; i=1,2,3,4,5,6$ instead of $d^{(i)} ; i=1,2,3,4,5,6$. All values from Table 1 have been taken as inputs in the imprecision form of $a_{i} \pm \delta_{i}$. This mathematical term about travel time components can be interpreted in the uncertain numeric statement type 'most possibly $t$ is between $a_{i}-\delta_{i}$ and $a_{i}+\delta_{i}$. Parameters of Gaussian membership function for seven travel time components are obtained using the aggregation procedure explained in earlier section. Combined group opinions, thus obtained are given in Table 5. Central value ' $a$ ' and deviation ' $\delta$ ' of Gaussian membership function is subsequently found out for total travel time with $\beta=2.0$. Travel time membership function with $\mu_{\tilde{T}} \rightarrow\{a=$ 84.8916; $\delta=5.9241\}$ is shown in Figure 4. Table 6 presents possibilistic reliability values computed for scheduled time range from 71 to 84.8916. It may be realized from Table 6 that scheduled time is crisp, as considered previously in subsection 5.1. Possibilistic reliability is zero for scheduled time of 71 and it goes on increasing and takes value of one at scheduled time equal to 84.8916 . 
International Journal of Mathematical, Engineering and Management Sciences

Vol. 6, No. 1, 223-243, 2021

https://doi.org/10.33889/IJMEMS.2021.6.1.014

Table 5. Gaussian membership function parameters of travel time components.

\begin{tabular}{|c|c|c|}
\hline \multirow{2}{*}{ Time Components } & \multicolumn{2}{|c|}{$\begin{array}{c}\text { Combined } \\
\text { Group Decision }\end{array}$} \\
\cline { 2 - 3 } & $a_{i}$ & $\delta_{i}$ \\
\hline$\tilde{T}_{p s h}$ & 3.5769 & 0.5262 \\
\hline$\tilde{T}_{w s h}$ & 1.4762 & 0.4364 \\
\hline$\tilde{T}_{n}$ & 61.8159 & 1.8203 \\
\hline$\tilde{T}_{d}$ & 9.1585 & 1.3252 \\
\hline$\tilde{T}_{w d h}$ & 4.0000 & 0.8165 \\
\hline$\tilde{T}_{d d h}$ & 2.0000 & 0.4082 \\
\hline$\tilde{T}_{c d h}$ & 2.8641 & 0.5912 \\
\hline
\end{tabular}

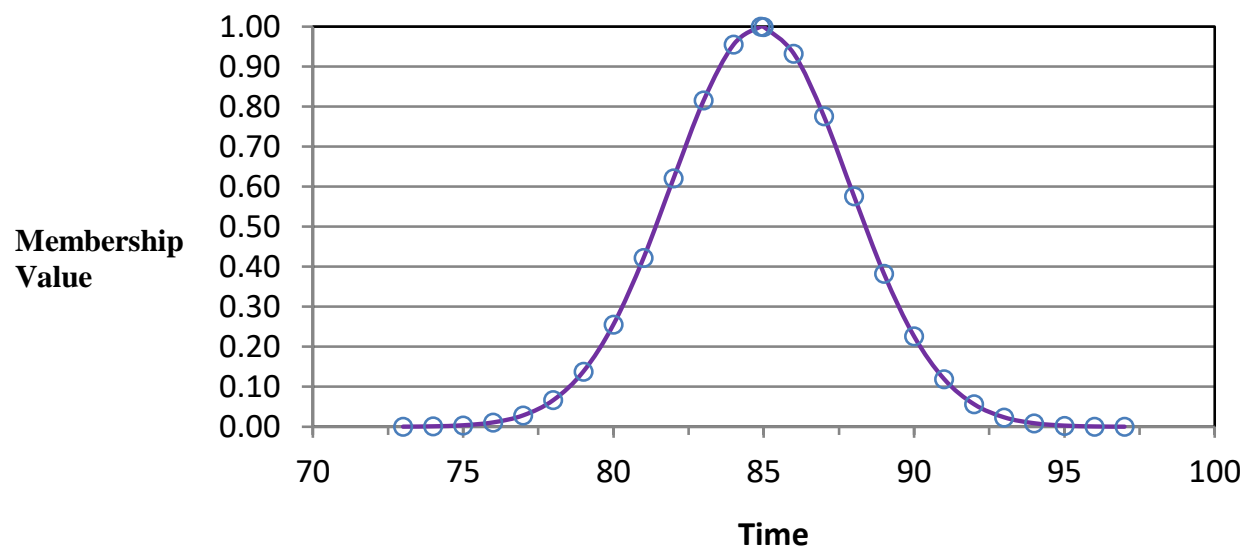

Figure 4. Gaussian membership function of travel time.

Table 6. Possibilistic reliability values (Third case).

\begin{tabular}{|c|c|}
\hline Scheduled Time & Possibilistic Reliability \\
\hline 71 & 0.0000 \\
\hline 72 & 0.0001 \\
\hline 73 & 0.0003 \\
\hline 74 & 0.0012 \\
\hline 75 & 0.0038 \\
\hline 77 & 0.0110 \\
\hline 78 & 0.0288 \\
\hline 79 & 0.0668 \\
\hline 80 & 0.1383 \\
\hline 82 & 0.2557 \\
\hline 83 & 0.4219 \\
\hline 84 & 0.6210 \\
\hline 848916 & 0.8155 \\
\hline & 0.9557 \\
\hline
\end{tabular}


International Journal of Mathematical, Engineering and Management Sciences

Vol. 6, No. 1, 223-243, 2021

https://doi.org/10.33889/IJMEMS.2021.6.1.014

\subsection{Sensitivity}

It is of interest to compute the possibilistic reliability of marine vessel for wide range of scheduled times that would help visualize the deviation and aid in ship's decision making. Possibilistic reliability variation for diverse scheduled times is depicted in Figure 5 for all three cases. TFN for travel time for first two is same; however, nature of right-hand side constraint variable of equation (5) is non-fuzzy and fuzzy in first two cases. In the first case, possibilistic reliability value for scheduled time of 71.5 is zero because TFN for travel time $\tilde{T}$ is fully on the right-hand side of scheduled time crisp constraint of 71.5. Possibilistic reliability then goes on increasing and becomes unity at scheduled time of 87.5. This is due to the fact that the mid value of $\tilde{T}$ tallies with scheduled time of 87.5 at which membership function value is maximum i.e. 1. When two fuzzy sets are compared, like in second case, possibility reliability end limits are obtained at different scheduled times. This owes to the fact that $t$ is having a possibilistic distribution here. When $\tilde{t}_{R}$ is equal to $\widetilde{T}_{L}$ i.e. at scheduled time of 71.5 , possibilistic reliability is 0 . But it may be noted that the scheduled time TFN at this stage is $\tilde{t}=[58.5,65,71.5]$ i.e. about 65 . Possibilistic reliability ascends later on and achieves a value of 1 when $\tilde{t}_{M}$ becomes equal to $\widetilde{T}_{M}$ at scheduled time of 87.5 . At this stage, scheduled time TFN is $\tilde{t}=[78.75,87.5,96.25]$. Readers are reminded that the fuzziness spread considered for $\tilde{t}$ is $10 \%$ in second case. To compare outcomes of first two cases, let scheduled time be $t=80$ (subsection 5.1) and about 80 i.e. $\tilde{t}=[72,80,88]$ (subsection 5.2). Corresponding possibilistic reliability values are $53.13 \%$ and $68.75 \%$ respectively. Higher value is attained in the second case due to the fact that the constraint variable is vague and having more imprecision in second case as against the first case. Moreover, though possibilistic reliability is $100 \%$ at same value in both the cases, the lower limit at which reliability values start increasing from $0 \%$ differ in both cases; due to reasons explained above. Higher reliability values in second case compared to first case is observed in Figure 5 at each scheduled time for the whole remaining in-between range.

Third case in subsection 5.3 is with Gaussian membership function for travel time and precise scheduled time variable constraint. The non-linear variation of possibilistic reliability can be noted from Figure 5. This can be attributed to very low membership values at extreme end of the Gaussian function to maximum value of 1 at the centre and the curve monotonically increases with high slope in-between. For $t=80$, possibilistic reliability in this case is $25.57 \%$. If possibilistic reliability variation in third case is gauged with other two cases, it is seen that initially its quite low compared to first two cases, but it raises steeply later and surpasses the corresponding values of other cases. For example, at $\tilde{t}_{M}$ and $t=77$, values of possibilistic reliability are 0.3438 (subsection 5.1), 0.5570 (subsection 5.2), 0.0288 (Subsection s.3) and at $\tilde{t}_{M}$ and $t=84$, values are $0.7813,0.8566$ and 0.9557 respectively.

It was concluded above that when fuzziness or imprecision exists in the scheduled time, possibilistic reliability takes a higher value. This was as per the assessment with precise scheduled time constraint. Fuzziness spread of $10 \%$ on each side of the constraint variable was considered in subsection 5.2. To confirm the above findings, sensitivity analysis is conducted. Imprecision with $5 \%$ and $15 \%$ spread is considered and the results are plotted in Figure 6 . All three variations take $100 \%$ reliability value at the scheduled time of 87.5 . This is due to the fact that both $\tilde{t}_{M}$ and $\widetilde{T}_{M}$ are mid values of respective TFN's and they turn out to be equal at scheduled time of 87.5 for all three spreads. The lower value at which possibilistic reliability take value equal to 0 differ for the reasons discussed before. Looking from the three variations of possibilistic reliability in Figure 6, it can be said that possibilistic reliability values appear higher as scheduled time fuzziness spread 
International Journal of Mathematical, Engineering and Management Sciences

Vol. 6, No. 1, 223-243, 2021

https://doi.org/10.33889/IJMEMS.2021.6.1.014

is wider.

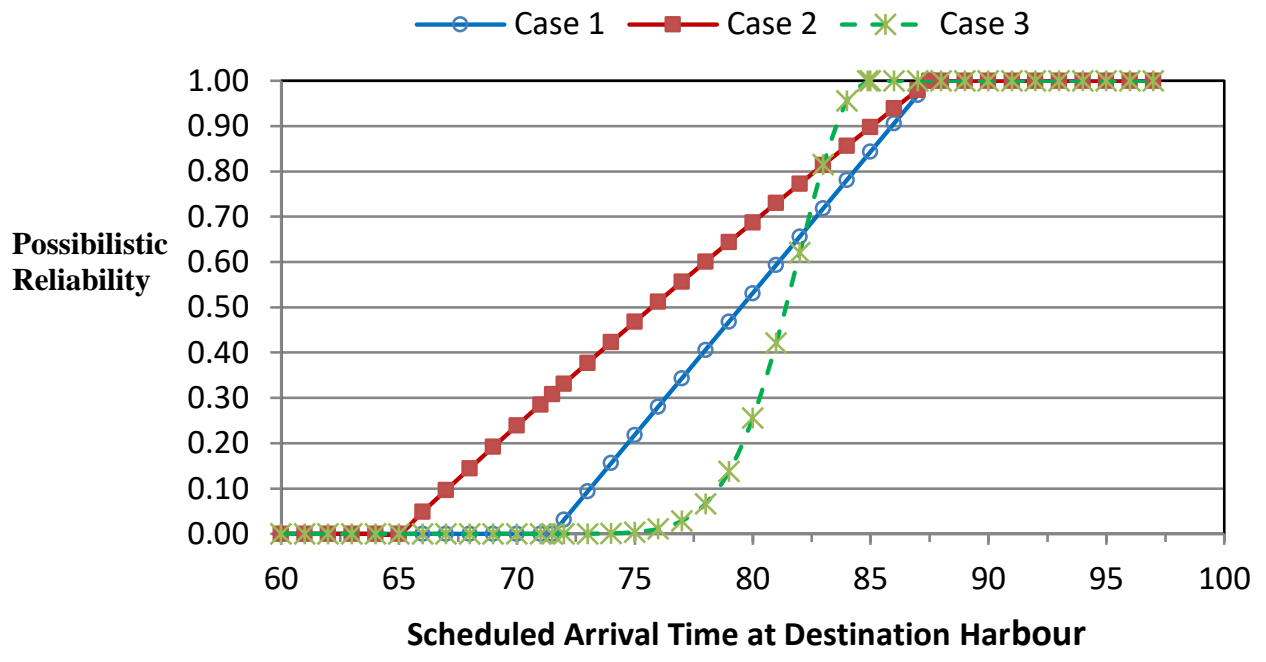

Figure 5. Variation of possibilistic reliability in all three cases.

Moreover, it is certainly of concern to study sensitivity of parameter $\beta$ in equation (8). This parameter decides the shape of possibilistic travel time distribution. Possibilistic reliability values change depending on the value of $\beta$ and thus extreme care must be taken while choosing this parameter. Sensitivity for various values of $\beta$ is shown in Figure 7. As, $\beta$ decides about fuzziness spread in the travel time, its value is decided by the membership function elicitation process by the analyst, as explained in Subsection 4.3. So, while deciding $\beta$, study of response behaviour of the expert group with regards to accuracy of their judgment or opinion is imperative.

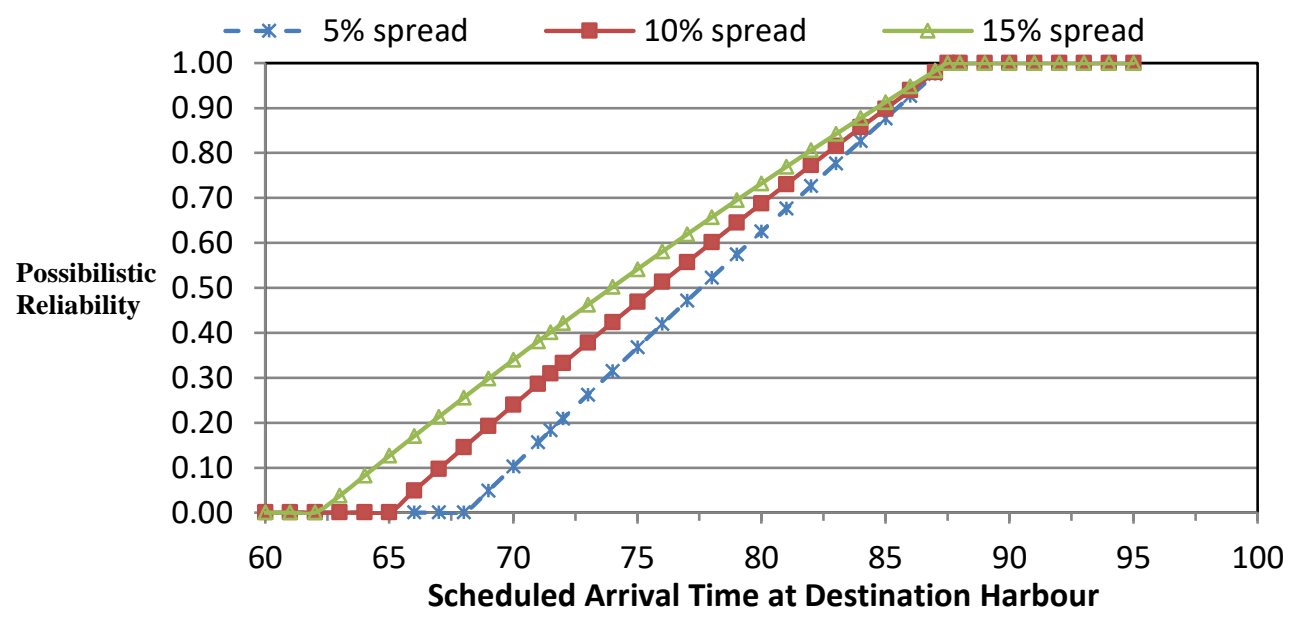

Figure 6. Sensitivity of fuzziness spread for second case. 
International Journal of Mathematical, Engineering and Management Sciences

Vol. 6, No. 1, 223-243, 2021

https://doi.org/10.33889/IJMEMS.2021.6.1.014

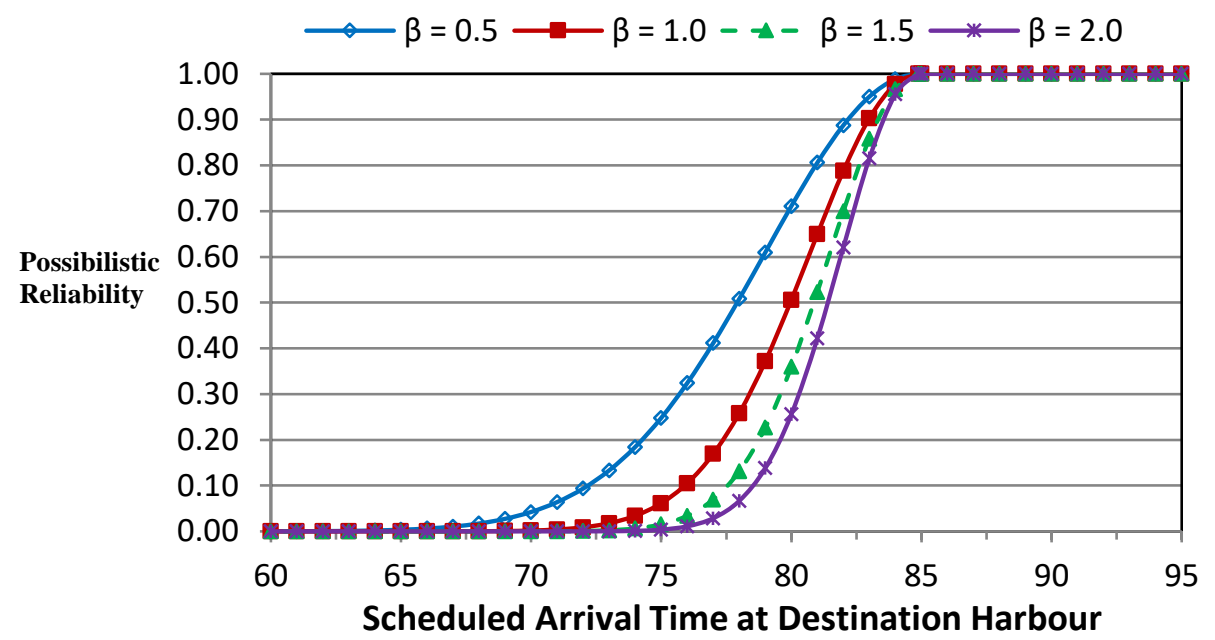

Figure 7. Sensitivity of parameter $\beta$ in third case.

It is also worthwhile to examine the effect of variation in fuzziness parameter $\beta$ of Gaussian membership function on travel time. Figure 8 shows the variation in travel time membership function for different values of fuzziness parameter i.e. $\beta=0.5,1.0,1.5,2.0,2.5$ and 3.0. As mentioned in earlier sections, analyst has to decide the suitable value of $\beta$ based on response behaviour of the experts and use of membership function elicitation methods. As seen from Figure 8 , the spread in membership function narrows down for higher $\beta$ values. This results in closer lower and upper bounds at any given $\alpha$-cut of the membership function and due to this, more accurate results of the reliability evaluation process are obtained.

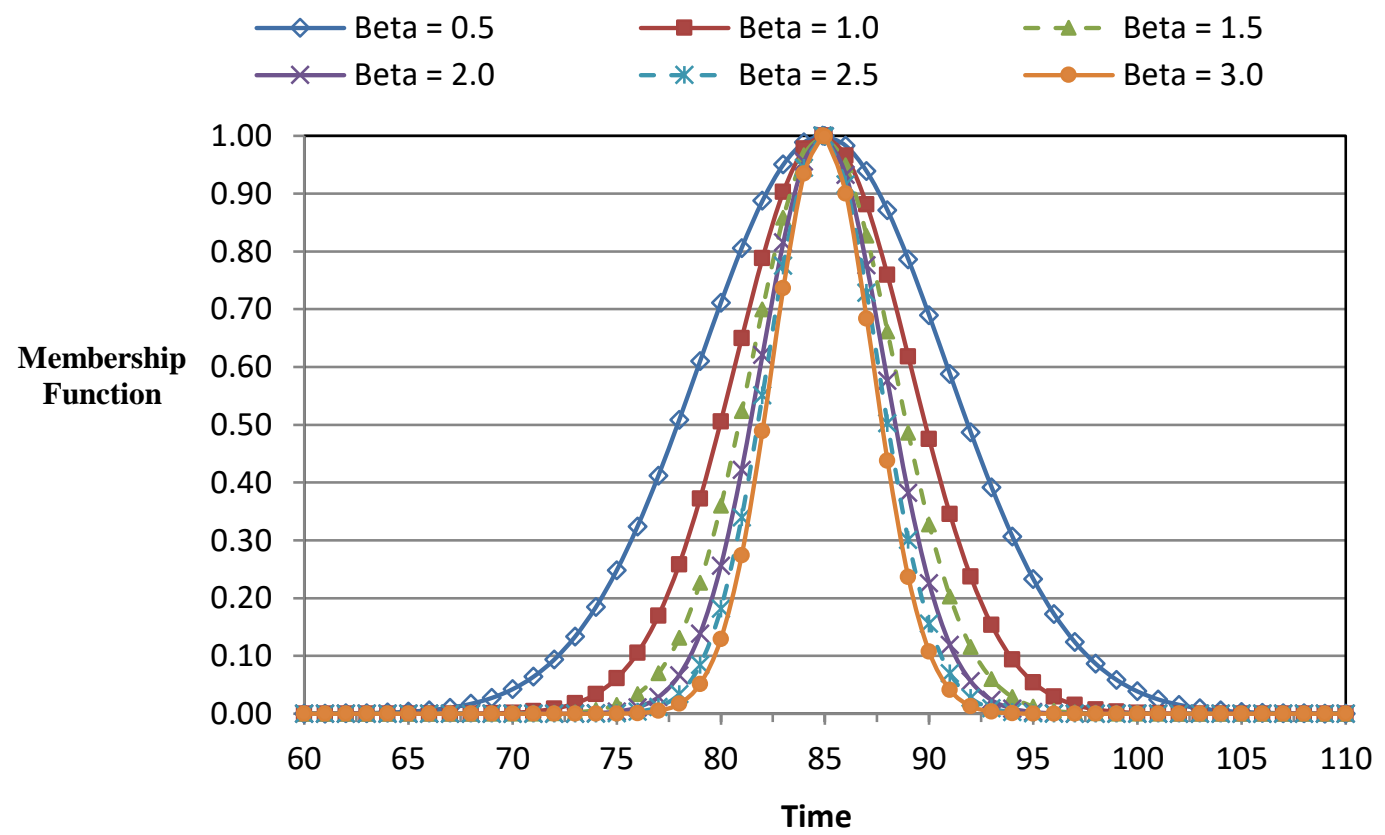

Figure 8. Effect of variation in $\beta$ on travel time. 
International Journal of Mathematical, Engineering and Management Sciences

Vol. 6, No. 1, 223-243, 2021

https://doi.org/10.33889/IJMEMS.2021.6.1.014

\subsection{Comments on the Cases}

Application of possibilistic concepts requires acquiring information from the experts in the problem domain under investigation, in our case, people working on marine vessel with vast experience at sea. The job of an analyst is to study the responses of the experts and elicit/ build suitable membership function with appropriate parameters for the travel time components. In illustration of first two cases, symmetric TFN is used. This is most suitable membership function when analyst is quite sure of opinions of the experts regarding their judgment about variable values. Furthermore, TFN is popular choice due to its linear characteristics, simplistic computation features and is used for fuzziness modelling on both sides of a single value of the variable. Gaussian membership function, used in the third case, has non-linear characteristics. This case considers expert opinions in imprecision form and requires comparatively difficult computation procedure. Some features of the illustrated cases are given in Table 7.

Table 7. Features of the three cases.

\begin{tabular}{|c|c|c|c|}
\hline Case & Case Application Requirement & Case Characteristics & Remarks \\
\hline I & $\begin{array}{l}\text { Needs domain expert's opinions for } \\
\text { acquiring travel time data } \\
\text { Scheduled time pre-planned or fixed }\end{array}$ & $\begin{array}{l}\text { Modelling with TFN } \\
\text { Comparison of TFN with non-fuzzy } \\
\text { value }\end{array}$ & Simplest model out of three \\
\hline II & $\begin{array}{l}\text { Needs domain expert's opinions for } \\
\text { acquiring travel time data and also the } \\
\text { scheduled time }\end{array}$ & $\begin{array}{l}\text { Modelling with TFN } \\
\text { Comparison of two TFN's }\end{array}$ & $\begin{array}{l}\text { Complex model compared to } \\
\text { Case I }\end{array}$ \\
\hline III & $\begin{array}{l}\text { Needs domain expert's opinions for } \\
\text { acquiring travel time data } \\
\text { Scheduled time pre-planned or fixed }\end{array}$ & $\begin{array}{l}\text { Modelling with Gaussian fuzzy number } \\
\text { Comparison of Gaussian fuzzy number } \\
\text { with non-fuzzy value }\end{array}$ & $\begin{array}{l}\text { Most Complex model } \\
\text { compared to other two }\end{array}$ \\
\hline
\end{tabular}

\section{Conclusion}

This paper presented an approach to compute travel time reliability of a transportation vehicle under fuzzy type of data. Travel time components have been identified in the beginning and then possibilistic reliability of transportation vehicle is defined. Unlike probabilistic approach, possibilistic approach takes care of uncertainties that are imprecise and vague. For this reason, it is used for modelling the travel time and related reliability. The proposed approach is demonstrated by a case of marine vessel carrying the bulk cargo between Incheon and Tokyo. Three different situations i.e. cases are formulated based on the nature of marine vessel's travel time and scheduled time of the vessel at the destination harbor. Possibilistic reliability computation procedure is illustrated for all three cases.

Later on, sensitivity analysis with regards to fuzziness in the scheduled time is carried out. It is concluded that fuzziness i.e. imprecision in the time values is a significant aspect in estimating the reliability precisely. As fuzziness in time variable is large, the range of uncertainty (imprecision) in scheduled time is more and it results in emergence of high possibilistic reliability value. In fact, fuzziness results in weaker constraints as against the precise or crisp one. This is quite logical as possibilistic theory deals with imprecision of different kind and naturally results in wider range or spread in constraint values.

Preference of individual case out of the three situations modelled in this paper depends upon the confidence of the experts regarding their judgment about variable values. If the experts are really sure of nature of their opinions, TFN could be used for modelling their responses. Otherwise, Gaussian membership function that models expert opinions in imprecision form is a sensible 
International Journal of Mathematical, Engineering and Management Sciences

Vol. 6, No. 1, 223-243, 2021

https://doi.org/10.33889/IJMEMS.2021.6.1.014

choice. Moreover, after well establishing the possibilistic reliability of any marine vessel over the time, it would also help in predicting the tentative travel time, scheduling the docking time of the vessel at the destination harbor, planning for the next vessel travel mission etc.

\section{Conflict of Interest}

The authors confirm that there is no conflict of interest to declare for this publication.

\section{Acknowledgement}

The authors would like to express their sincere thanks to the editor and anonymous reviewers for their time and valuable suggestions.

\section{References}

Al-Deek, H.M., \& Emam, E.B. (2006). Computing travel time reliability-in transportation networks with multistates and dependent link failures. Journal of Computing in Civil Engineering, 20(5), 317-327.

Batley, R. (2007). Marginal valuations of travel time and scheduling, and the reliability premium. Transportation Research Part E-Logistics and Transportation Review, 43(4), 387-408.

Bell, M.G.H. (2000). A game theory approach to measuring the performance reliability of transport networks. Transportation Research Part B-Methodological, 34(6), 533-545.

Cai, K.Y. (1996). Introduction to fuzzy reliability. Springer, Boston, MA. USA.

Chang, J.S. (2010). Assessing travel time reliability in transport appraisal. Journal of Transport Geography, $18(3), 419-425$

Chen, Z., \& Fan, W. (2020). Analyzing travel time distribution based on different travel time reliability patterns using probe vehicle data. International Journal of Transportation Science and Technology, 9(1), $64-75$.

Clark, S., \& Watling, D. (2005). Modelling network travel time reliability under stochastic demand. Transportation Research Part B-Methodological, 39(2), 119-140.

Ding, Y., \& Lisnianski, A. (2008a). Fuzzy universal generating functions for multi-state system reliability assessment. Fuzzy Sets and Systems, 159(3), 307-324.

Ding, Y., Zuo, M.J., Lisnianski, A., \& Tian, Z. (2008b). Fuzzy multi-state system: general definition and performance assessment. IEEE Transactions on Reliability, 57(4), 589-594.

El Faouzi, N.E., Billot, R., \& Bouzebda, S. (2010). Motorway travel time prediction based on toll data and weather effect integration. IET Intelligent Transportation Systems, 4(4), 338-345.

Ettema, D., \& Timmermans, H. (2006). Costs of travel time uncertainty and benefits of travel time information: Conceptual model and numerical examples. Transportation Research Part C-Emerging Technologies, 14(5), 335-350.

Fan, Y.Y., \& Nie, Y. (2006). Optimal routing for maximizing the travel time reliability. Networks \& Spatial Economics, 6(3-4), 333-344.

Fosgerau, M., \& Karlstrom, A. (2010). The value of reliability. Transportation Research Part BMethodological, 44(1), 38-49.

Gaonkar, R.S.P., Xie, M., \& Fu, X. (2013b). Reliability estimation of maritime transportation: a study of two fuzzy reliability models. Ocean Engineering, 72, 1-10. 
International Journal of Mathematical, Engineering and Management Sciences

Vol. 6, No. 1, 223-243, 2021

https://doi.org/10.33889/IJMEMS.2021.6.1.014

Gaonkar, R.S.P., Xie, M., Ng, K.M., \& Habibullah, M.S. (2011). Subjective operational reliability assessment of maritime transportation system. Expert Systems with Applications, 38(11), 13835-13846.

Ghader, S., Darzi, A., \& Zhang, L. (2019). Modeling effects of travel time reliability on mode choice using cumulative prospect theory. Transportation Research Part C: Emerging Technologies, 108, 245-254.

Halgamuge, S.K., Poechmueller, W., \& Glesner, M. (1995). An alternative approach for generation of membership functions and fuzzy rules based on radial and cubic basis function networks. International Journal of Approximate Reasoning, 12(3-4), 279-298.

Hollander, Y., \& Liu, R.H. (2008). Estimation of the distribution of travel times by repeated simulation. Transportation Research Part C-Emerging Technologies, 16(2), 212-231.

Huang, H.Z., \& Zhang, X.D. (2009). Design optimization with discrete and continuous variables of aleatory and epistemic uncertainties. Journal of Mechanical Design, 131(3), 031006-031001-031006-031008.

Innamaa, S. (2005). Short-term prediction of travel time using neural networks on an interurban highway. Transportation, 32(6), 649-669.

Inuiguchi, M., \& Ramik, J. (2000). Possibilistic linear programming: a brief review of fuzzy mathematical programming and a comparison with stochastic programming in portfolio selection problem. Fuzzy Sets and Systems, 111(1), 3-28.

Inuiguchi, M., Tanino, T., \& Sakawa, M. (2000). Membership function elicitation in possibilistic programming problems. Fuzzy Sets and Systems, 111(1),29-45.

Iskander, M.G. (2002). Comparison of fuzzy numbers using possibility programming: comments and new concepts. Computers \& Mathematics with Applications, 43(6-7), 833-840.

Janic, M. (2003). Modelling operational, economic and environmental performance of an air transport network. Transportation Research Part D-Transport and Environment, 8(6), 415-432.

Jiang, Q.M., \& Chen, C.H. (2003). A numerical algorithm of fuzzy reliability. Reliability Engineering \& System Safety, 80(3), 299-307.

Klir, G.J., Clair, U.S., \& Yuan, B. (1997). Fuzzy set theory: foundations and applications. Prentice Hall. USA.

Kreinovich, V., Quintana, C., \& Reznik, L. (1992, December). Gaussian membership functions are most adequate in representing uncertainty in measurements. In Proceedings of NAFIPS (Vol. 92, pp. 15-17).

Kwong, K., Kavaler, R., Rajagopal, R., \& Varaiya, P. (2010). Real-time measurement of link vehicle count and travel time in a road network. IEEE Transactions on Intelligent Transportation Systems, 11(4), 814825 .

Li, B. (2019). Measuring travel time reliability and risk: A nonparametric approach. Transportation Research Part B: Methodological, 130, 152-171.

Li, B., Zhu, M.L., \& Xu, K. (2000). A practical engineering method for fuzzy reliability analysis of mechanical structures. Reliability Engineering \& System Safety, 67(3), 311-315.

Li, H., He, F., Lin, X., Wang, Y., \& Li, M. (2019). Travel time reliability measure based on predictability using the Lempel-Ziv algorithm. Transportation Research Part C: Emerging Technologies, 101, 161180.

Li, Z., Hensher, D.A., \& Rose, J.M. (2010). Willingness to pay for travel time reliability in passenger transport: A review and some new empirical evidence. Transportation Research Part E-Logistics and Transportation Review, 46(3), 384-403. 
International Journal of Mathematical, Engineering and Management Sciences

Vol. 6, No. 1, 223-243, 2021

https://doi.org/10.33889/IJMEMS.2021.6.1.014

Liu, Y., Huang, H.Z., \& Levitin, G. (2008). Reliability and performance assessment for fuzzy multi-state elements. Proceedings of the Institution of Mechanical Engineers, Part O: Journal of Risk and Reliability, 222(4), 675-686.

Marano, G.C., \& Quaranta, G. (2010). A new possibilistic reliability index definition. Acta Mechanica, 210(3-4), 291-303.

Margulici, J.D., \& Ban, X. (2008). Benchmarking travel time estimates. IET Intelligent Transport Systems, 2(3), 228-237.

Medasani, S., Kim, J., \& Krishnapuram, R. (1998). An overview of membership function generation techniques for pattern recognition. International Journal of Approximate Reasoning, 19(3), 391-417.

Nam, D., Park, D., \& Khamkongkhun, A. (2005). Estimation of value of travel time reliability. Journal of Advanced Transportation, 39(1), 39-61.

Negi, D.S., \& Lee, E.S. (1993). Possibility programming by the comparison of fuzzy numbers. Computers \& Mathematics with Applications, 25(9),43-50.

Nicholson, A., \& Dantas, A. (2004). Proceedings of the Second International Symposium on Transportation Network Reliability (INSTR). New Zealand, 139-144.

Noland, R.B., \& Polak, J.W. (2002). Travel time variability: a review of theoretical and empirical issues. Transport Reviews, 22(1), 39-54.

Noland, R.B., Small, K.A., Koskenoja, P.M., \& Chu, X.H. (1998). Simulating travel reliability. Regional Science and Urban Economics, 28(5), 535-564.

Pedrycz, W., \& Vukovich, G. (2002). On elicitation of membership functions. IEEE Transactions on Systems, Man, and Cybernetics-Part A: Systems and Humans, 32(6), 761-767.

Prabhu Gaonkar, R.S., Xie, M., \& Huang, H.Z. (2013a). Optimizing maritime travel time reliability. Proceedings of the Institution of Mechanical Engineers, Part M: Journal of Engineering for the Maritime Environment, 227(2), 167-176.

Reventós, V.T.I. (1999). Interpreting membership functions: A constructive approach. International Journal of Approximate Reasoning, 20(3), 191-207.

Saedi, R., Saeedmanesh, M., Zockaie, A., Saberi, M., Geroliminis, N., \& Mahmassani, H.S. (2020). Estimating network travel time reliability with network partitioning. Transportation Research Part C: Emerging Technologies, 112, 46-61.

Sanchez-Silva, M., Daniels, M., Lleras, G., \& Patino, D. (2005). A transport network reliability model for the efficient assignment of resources. Transportation Research Part B: Methodological, 39(1), 47-63.

Senna, L.A. (1994). The influence of travel time variability on the value of time. Transportation, 21(2), 203228.

Sumalee, A., \& Kurauchi, F. (2006). Guest editorial: reliability and emergency issues in transportation network analysis. Networks and Spatial Economics, 6(3-4), 169.

Tzannatos, E.S. (2005). Technical reliability of the Greek coastal passenger fleet. Marine Policy, 29(1), 8592.

van Lint, J.W.C. (2008). Online learning solutions for freeway travel time prediction. IEEE Transactions on Intelligent Transportation Systems, 9(1), 38-47.

van Lint, J.W.C., van Zuylen, H.J., \& Tu, H. (2008). Travel time unreliability on freeways: why measures based on variance tell only half the story. Transportation Research Part A: Policy and Practice, 42(1), 258-277. 
International Journal of Mathematical, Engineering and Management Sciences

Vol. 6, No. 1, 223-243, 2021

https://doi.org/10.33889/IJMEMS.2021.6.1.014

Vanajakshi, L., Subramanian, S.C., \& Sivanandan, R. (2009). Travel time prediction under heterogeneous traffic conditions using global positioning system data from buses. IET Intelligent Transport Systems, 3(1), 1-9.

Verma, A.K., Srividya, A., \& Gaonkar, R.S.P. (2006). Deciding dynamic inspection frequency: A fuzzy set approach. International Journal of Performability Engineering, 4(1), 45-59.

Wu, H.C. (2004). Bayesian system reliability assessment under fuzzy environments. Reliability Engineering \& System Safety, 83(3), 277-286.

Wu, W.W., Ning, A., \& Ning, X.X. (2008). Evaluation of the reliability of transport networks based on the stochastic flow of moving objects. Reliability Engineering \& System Safety, 93(6), 838-844.

Wu, X., Roy, U., Hamidi, M., \& Craig, B.N. (2020). Estimate travel time of ships in narrow channel based on AIS data. Ocean Engineering, 202, 106790.

Yang, M., Liu, Y., \& You, Z. (2009). The reliability of travel time forecasting. IEEE Transactions on Intelligent Transportation Systems, 11(1), 162-171.

Yu, S.C., \& Wu, B. (2009). Fuzzy item response model: a new approach to generate membership function to score psychological measurement. Quality and Quantity, 43(3), 381.

Zhang, X., \& Huang, H.Z. (2010). Sequential optimization and reliability assessment for multidisciplinary design optimization under aleatory and epistemic uncertainties. Structural and Multidisciplinary Optimization, 40(1-6), 165.

Zheng, P., \& McDonald, M. (2009). Estimation of travel time using fuzzy clustering method. IET Intelligent Transport Systems, 3(1), 77-86. 